\title{
Perceived Barrier in Accessing Emergency Medical Services of Ethnic Groups in the Highlands of Chiang Rai Province, Thailand
}

Songwut Sungbun, M.N.S., Thitiarpha Tangkawanich, Ph.D., Orruethai Thanakumma, M.N.S., Alongkorn Sukrueangkul, M.N.S.

Faculty of Nursing, Chiang Rai College, Mueang, Chiang Rai 57000, Thailand.

Received 20 June 2019 • Revised 18 September 2019 • Accepted 13 January 2020 • Published online 26 March 2020

\section{Abstract:}

Objective: To explore the barriers of accessing emergency medical services among several ethnic groups, including the Hmong, Lisu, Lahu and Akha people.

Material and Methods: The qualitative study was conducted to interview 43 participants, including the Akha, Lahu, Lisu and Hmong people. Purposive sampling was used to select participants for in-depth interviews, from February to March, 2019. The data were analyzed using content analysis.

Results: The results show that the barriers of using emergency medical service (EMS) can be divided into the following five categories: the barrier to effectively communication, response to the symptoms of emergency illnesses, transportation facilities to support patients with emergency illnesses, fear to contact healthcare providers and the individual's perception of emergency medical symptoms.

Conclusion: The barriers of using EMS among different cultures, ethnicities and languages are a concern and obstacles to the increase appropriate pre-hospital management and reducing pre-hospital delay. A community collaboration partnership should be the goal of improving pre-hospital care among ethnic people.

Keywords: barriers in accessing EMS, emergency medical service, ethnic group, highland

Contact: Songwut Sungbun, M.N.S.

Faculty of Nursing, Chiang Rai College, Mueang, Chiang Rai 57000, Thailand.

E-mail: Songwut.Sungbun@crc.ac.th

() 2020 JHSMR. Hosting by Prince of Songkla University. All rights reserved.

This is an open access article under the CC BY-NC-ND license

(http://www.jhsmr.org/index.php/jhsmr/about/editorialPolicies\#openAccessPolicy).
J Health Sci Med Res 2020;38(2):125-133 doi: 10.31584/jhsmr.2020731 www.jhsmr.org 


\section{Introduction}

Emergency illnesses are sudden and life-threatening conditions that constitute serious health problems, and the time to definitive care influences the clinical outcomes of emergency patients. The emergency medical service (EMS) system provides professional pre-hospital emergency medical care for timely management between the outhospital and emergency department. ${ }^{1-3}$ Although the use of EMS for transportation shows an improving survival rate, when compared with the use of private vehicles ${ }^{2,3}$, the number of emergency patients who prefer EMS for transportation is limited. ${ }^{4}$ National Institutes of Emergency Medicine Thailand (NIEM) regulate the policy of emergency medical services to promote equity and quality of services. ${ }^{4}$ However, there were over 20 million emergency patients who visited the emergency departments of government hospitals in 2011, while those using EMS for transportation only accounted for 860,000 of this number. ${ }^{4}$ There were $80.6 \%$ of emergency patients who used self-transportation; wherein, only $19.1 \%$ used EMS for transportation. Among EMS usage, $70.1 \%$ were non-trauma patients, with $29.9 \%$ making up trauma patients. ${ }^{4}$ The results showed that emergency patients did not have an understanding in regards to EMS, or knew the EMS telephone number (1669) in case of a medical emergency. Moreover, there was a lack of knowledge about cases of medical emergencies; such as, acute myocardial infarction (MI), acute stroke and trauma that require management within a short time for an effective outcome. , $3,5^{2}$ Unfortunately, the majority of patients with acute $\mathrm{Ml}$ and acute stroke used a private vehicle for transportation, while the international guidelines of the stroke and acute $\mathrm{Ml}$ association recommended patients use EMS for transportation. ${ }^{5}$

Many communities who need EMS for transportation in Thailand are located far away from healthcare services. Although, the national policy of EMS was formulated for equity and quality of service, the strategies might not be implemented effectively, due to geographical difficulties. ${ }^{6,7}$ Thailand has a wide range of geographical landscapes, characterized by several mountain ranges, especially in northern Thailand. Several ethnic groups migrated from South China to Thailand, and now live in the five provinces of Thailand that consist of; Chiang Rai, Chiang Mai, Tak, Phrae and Lampang. ${ }^{8}$ These ethnic people are divided into six groups that include the Akha, Lahu, Lisu, Hmong, Yao and Karen people. ${ }^{9}$ Chiang Rai province has several ethnic groups who live in the highlands at a greater distance from medical services. Additionally, there are different languages, cultures and lifestyles among the different ethnic groups that influence healthcare practices. ${ }^{8-11}$ However, they have a culture of sharing information that has a positive impact on health issues, and this has led to a challenge in the promotion of equity and qualities of care. ,10,12,13 $^{9,13}$

Previous studies have shown that public knowledge of using EMS is important for an increment of patients using EMS for transportation. While no study focuses on an individual's perception of using EMS, based on cultural diversity and values, understanding cultural diversity instead of individuals would be an important component; allowing for specific strategies of pre-hospital care. This study aimed to explore an individual's perception of the barriers that are associated with using EMS among several ethnic groups. The information of the perceived barriers for using EMS among cultural diversities in Thailand will enable healthcare providers to formulate strategies of care and promote effective pre-hospital care.

\section{Material and Methods}

This study was approved by the Chiang Rai Provincial Public Health Board (Code: CRPPHO 11/ 2562). Interviews were performed after the participants gave written informed consent. 
This study focused on several ethnic groups who live in the Mea Saruay district, Chiang Rai province in Thailand. Purposive sampling was used to select the participants, who met the following criteria: (1) aged 20-65 years old, (2) ethnicity including Hmong, Lisu, Lahu and Akha groups, and (3) voluntarily participated in this study. There were 43 participants, including $10 \mathrm{Hmong}$ people, 10 Lisu people, 10 Lahu people and 13 Akha people who volunteered for an in-depth interview in this study.

This qualitative study was conducted with face-toface interviews, and was performed in the Thai language by the researcher. The interview guide was developed from a pilot study of 15 ethnic people who had experience using EMS for transportation, so as to ensure obvious understanding of the interview guide. The interview guide was validated by three experts, and the content validity index was 0.90. The guide were used during audiorecorded interviews, while for participants who could not speak Thai the questions were translated into the local language of the participants by interpreters. The interpreters translated questions to obtain information from the participants in real-time conversations. ${ }^{14}$ There were four interpreters, who have been trained knowledge in, strokes and myocardial infraction disease. The interpreters had experience with using EMS for transportation, and could speak both the Thai language and local languages of the ethnic groups, such as Hmong, Lisu, Lahu or Akha. The interpreters' role was to translate word by word during the interview. ${ }^{14,15}$ The time spent on interview completion was approximately 20 minutes. The audio-record was transcribed verbatim, line by line by the researcher and checked for precision by two co-researchers. The findings were then back-translated from the Thai language to the Hmong, Lisu, Lahu and Akha languages, by the translators to ensure accuracy with 6 participants.

Four strategies have been proposed to ensure the trustworthiness of this study, including credibility, trans- ferability, dependability and confirmability (conformability). ${ }^{16}$ For credibility of these findings a triangulation method was used: interviews, filed notes, and observation. To achieve dependability, the researchers used colleagues and methodological experts to check the research plan and research methodology. The confirmability (conformability) was achieved through the audit method of data analysis, filed notes as well as observation findings that were checked and rechecked by the research team. ${ }^{16}$ The findings presented in the source language are being preserved in the target language, after the translation for confirmability (conformability), then researchers discussed with an experienced, qualitative expert who was bilingual (Thai and ethnic languages). ${ }^{15}$

The data were analyzed using content analysis..$^{14,15,17}$ Two researchers re-checked verbatim transcriptions from each participant, and reviewed the transcripts with audiorecords several times in order to ensure the data was correct. The content of the data was coded into key findings, that were categorized into subcategories, line by line based on similar meaning and phenomena. The subcategories were categorized into categories, following translation of all themes and extracts into English. ${ }^{17}$

\section{Results}

The majority of participants were women (83.4\%), $16.6 \%$ were men. The average age was 47.5 years old. Half of the participants had a primary school education, with $41.9 \%$ being informally educated. After analyzing the interviews, regarding the perception of symptom management during medical emergency conditions, the participants identified their perceptions into five categories: Barrier of effective communication, response to the symptoms of emergency illnesses, transportation facilities to support patients with emergency illnesses, fear of contacting healthcare providers and the individual's perception of emergency medical symptoms. 


\section{The barrier to effective communication}

Some of the participants emphasized that most of the people in their village were unable to speak or understand the Thai language. As a large number of participants were unble to speak the Thai language fluently coupled with local healthcare providers being are unable to understand the patient's language was one barrier of healthcare delivery; hence translators were important when they contacted healthcare providers.

"The elderly in my village cannot speak the Thai language and do not understand the Thai language... when they need to see a doctor, they need someone to help translate.” (Participant no. 22)

"Sometimes when I see a doctor, I understand what he said to me but he does not understand what I say to him... I try to say it again and again... My grandmother, she was unable to understand Thai, when I take her to a hospital, I want a translator too" (Participant no. 24)

Some of the participants agreed on the need for translators, while seeking healthcare services, whilst other participants mentioned they wait for family members, who can speak and understand Thai, to take them to hospital.

"I cannot go to the hospital alone, because I do not understand Thai... If I have a serious health condition (severe headache and difficulty breathing), I have to wait for my son (he can speak and understand the Thai language) to come back from school." (Participant no. 40)

"When I went to the hospital, all of the healthcare providers did not understand what I said... when I need to go to the hospital, I wait for my daughter who can speak Thai... she is a translator." (Participant no. 38)

Most of the health information provided in hospitals, and on medicine labels was in Thai. The participants reported that they did not understand how to use the medicine, nor information about giving and taking a drug. Some participants believed that pictures or symbols might help them understand the medical labels.
"I have some problem with my heart, and I took this medicine (she showed warfarin tablets to the researchers)... I forgot to take it, because I cannot read medical labels. I have to take it which days... when I saw the doctor again, the pharmacist described how to take this medicine again, and used the symbols that represented day and time...this was very helpful." (Participant no. 18)

\section{Response to symptoms of emergency illness}

A large group of the participants did not have knowledge in reqards to information about emergency illnesses, the telephone number of EMS or self-management in response to medical emergency conditions. Knowledge about symptoms of emergency illnesses is important for people making decisions in seeking healthcare providers; however they do not seek healthcare services until they have severe signs and symptoms as well as severe conditions.

"I do not know about emergency illnesses, or the phone number of the ambulance service... when I get ill, I try to use the traditional medicine first." (Participant no. 6) "I did not know what are stroke and Ml symptoms... I did not know what is an appropriate time to see the doctor after having the symptoms of stroke and MI." (Participant no. 30)

Participants presented that they used traditional medicine during emergency symptoms, such as chest pain. They seek traditional medicine to treat their symptoms, because they believe traditional medicine can reduce any symptom.

"Sometimes when I get ill... a traditional healer said to me that I should pray for a ghost, because it made me discomfort and after I did, then I felt better... । believed that a ghost can help me" (Participant no. 3)

"When I had chest pain... I used Gua-Sa (this is a traditional treatment, performed by a traditional healer), after I received it, I had bruises on my skin) after that I 
went to the community hospital... when I get sick, I use traditional medicine every time and then I always feel better." (Participant no. 18)

\section{Transportation facilities to support patients} with emergency illness

A large portion of participants perceived the need for an ambulance for transportation, and medical equipment in the ambulance during transportation. The participants were also concerned about patient safety during both self-transportation and using EMS for transportation. Most participants were aware of the vehicle for transporting patients to hospitals, because all of them did not have their own vehicle, especially a car.

"When my mother had severe diarrhea, it was very difficult to take her to the community hospital, because I did not have my own car... I tried to request a car from my neighbors, but I felt like an embarrassment when I contacted my neighbors in the night time... I felt unsafe, because the car did not provide any medical equipment, such as an oxygen tank.” (Participant no. 31)

"When we get ill, we go to a sub-district hospital first... if we have severe symptoms, we are referred to a community hospital by a car of the sub-district health promoting hospital... sometimes if the car is not available, we go to community hospital by private vehicle.” (Participant no. 18)

The characteristics of road and traffic lights were disruptive for patients traveling to the hospital, because the drivers drive badly in a car or motorcycle at night time. They reported that during the rainy season it is difficult to use any vehicle.

"In the rainy season, it is very difficult to use a motorcycle for transporting patients to the community hospital, because it is unsafe; the road in my village is very dangerous when it is raining... at night it is very dark, and we have to drive our car slowly." (Participant no. 24)
In general, a car was provided by the sub-district Health Promoting Hospitals during office hours in case of medical emergency conditions, while during non-office hours they used a private vehicle. All of the participants reported the cost of transportation to be about 1,000-1,500 baht, per time.

"I paid for private transportation, about 1,000 to 1,500 baht each time... although it is expensive, we did not have another choice." (Participant no. 18)

"I do not have my own car, when I or my family members get sick. we try to take the old medicines; which were received from the hospital in the past... we cannot go to the hospital immediately because we cannot pay the transportation cost." (Participant no. 23)

\section{Fear to contact healthcare providers}

Some of the participants did not receive any class of Thai citizenship. As a result, many participants, who are migrants, fear contact with the local government and healthcare providers. Sometimes they received medical services from local, private medical care providers.

"I fear to see the doctor, because I do have not a Thai identity card... I have lived in Thailand since I was young, for about 50 years, but I have never had any contact with government officials." (Participant no. 30)

"Some of the members of my village are not Thai citizens... some of them received a non-citizen card from the local government, but there are some limitations on healthcare services. They do not want to see a modern doctor in a hospital." (Participant no. 37)

Thai citizens receive identity cards indicating eligibility for Universal Health Coverage (UC) insurance, while some of the participants had a class of non-citizenship identification cards. Some of the participants did not have health insurance, especially UC Insurance. They fear contacting healthcare providers because they are concerned about the cost of service. 
"I do not have any health insurance. When I need to see a doctor, I fear to contact them (healthcare providers) ... because I have to pay for medical services... I try to use traditional medicine because I do not have much money to pay for modern medicine." (Participant no. 11)

\section{Individual's perception of emergency medical} symptoms

Some of the participants reported that emergency illnesses, such as acute stroke and acute $\mathrm{Ml}$ were not important. They believed that healthy people cannot have an acute stroke and acute Ml. Some of the participants perceived the wrong information about emergency illnesses from their experiences.

"I heard about (acute stroke and acute MI) from television... I am healthy. I have no fear about this... my neighbor had a stroke, and after two days he went to the hospital. Now I see that he is healthy, after he got the medicine.” (Participant no. 12)

“Last month when I met my doctor to follow up my hypertension, the doctor talked about stroke and MI, but now I forgot it... now my blood pressure is normal and I am healthy. I do not have any health problems." (Participant no. 22)

\section{Discussion}

In Thailand, several ethnic people have migrated from many counties from the northern areas of Asia. They have their own identifiable culture, lifestyle, behavior and language, which are related to public health quality in Thailand, especially emergency medical care. In regards to the language barrier, the low literacy rate is a vital culprit. ${ }^{10,18}$ Several studies have shown that language is a major problem in accessing healthcare services and health information among several ethnic groups in Thailand., 9,,19 Moreover, previous studies reported that the language barrier has resulted in misdiagnosis, treatment delay and poor prognosis in the emergency department. ${ }^{9,-11,20-22}$ The language abilities might be disparities of need in concern to health information among different ethnic people., , 10,22 $^{2}$ Furthermore, most of those groups did not have knowledge about how to access EMS, while NIEM had the telephone number of EMS through television promotion and advertisements. Reasonably, this scenario might reflect the low perception and awareness of ethnic people, who use different languages in Thailand. Non-effective communication has been a cause for a reduced use of preventative services ${ }^{23}$, EMS and poor health outcomes; such as an increased hospitalization rate as well as mortality. ${ }^{13,24}$ The 1669 application cannot promote this area, because of complications arising due to language ability and understand the difficulty in its usage. Local healthcare providers should focus on young people as translators, and volunteers in a healthcare setting, in addition to training them as health promoters, because most of them can speak and understand both Thai and the patients' language. ${ }^{9}$

The barrier of communication is not only about language abilities, but also the lack of any mobile phone signal, or very weak fringe reception, in some highland area. Poor mobile phone signals from the network was another reason for the lack of EMS usage. Use of a radio operation within EMS systems might be addressed for communication in areas where there is a lack of any mobile phone signal. Therefore, promoting local healthcare providers and leaders in communities in the usage of radio operation, for communication between dispatch centers and local healthcare services, should also be addressed. It is justifiable to improve public knowledge about the response to medical emergency conditions along with mobile signal networks.

Culture and experience may play a vital role in an individual's perception of responding to emergency illnesses. ${ }^{9,10}$ In this study, the Akha people needed information on decision-making, and response to symptoms 
in case of emergency illness, when compared with other ethnic groups. They believe that traditional medicine can alleviate any illness. Hence, this leads to inappropriate management for emergency illnesses. ${ }^{11}$ Use of traditional medicine might be as a result of delayed decision making to seek healthcare service, and treatment delay. Awareness of appropriate responses to emergency illnesses can be high or low, depending on the attention given to ethnic groups. ${ }^{21} \mathrm{~A}$ possible solution may be using social influence transmitted information of response to emergency illness, including the village headman, local healthcare workers and respected persons in the community, so as to enhance effective communication, empathy and trust; while giving health information. The strategies should encourage community collaborative partnerships to work together with local healthcare workers to change personal perceptions of health..$^{10,25}$

Transportation is another factor that influences pre-hospital management of patients with an emergency illness. $^{6,27}$ Transportation facilities were acknowledged among all of the ethnic groups, because they did not have their own car. Furthermore, the elderly in the study area needed their family members to be supportive of their transportation needs, by taking them to receive medical services, while their family members worked outside their village. The barriers of communication, use of mobile phone abilities, and traditional lifestyle influence the scenarios of using EMS for transportation among elderly people in this area. This current study showed that elderly people in rural areas in Thailand need to be supported with transportation; someone must take them to hospital. ${ }^{4-6}$

However, the problem of financial support, during medical emergency conditions, is also a major problem that affects time to treatments..$^{9,19,20,27}$ The majority of participants complained about the cost of transport, cost of healthcare services and medicine. They stated that these are the reasons for delayed treatment, and non- use of medical services. Some of them are migrants, who do not have universal coverage insurance, leading to a lack of use of healthcare services. Some of them who had Thai citizenship status reported that they generally were able to access basic healthcare services. The poor economic conditions, households with a low income as well as no health insurance are an obstacle and limitation to accessing healthcare delivery, causing treatment delays. ${ }^{11,19,20,26,28}$ The holistic professional approach to the health of migrant people into mainstream healthcare practices, health policy, and local political policy should lead to promoting equity and quality of healthcare service.

This study had some limitations. Most of the participants did not have any experience using EMS for transportation, because the number of people using EMS for transportation in this area is limited. Some of the participants cannot speak or understand the Thai language, but they were able to fill in the gap of knowledge in this study.

\section{Conclusion}

The characteristics of geographical terrain, and the different characteristics of the people with different local cultures and languages are the factors that challenge healthcare providers in developing an EMS system around Thailand. Although, language abilities and different cultures from the majority of people are a serious barrier to promoting EMS, a multidisciplinary team approach; including local government, healthcare providers, teachers, social development workers and leaders in communities along with community collaboration could destroy both the barriers to assessing EMS and healthcare services among several ethnic groups. There is a need for improving awareness and perception of fast track stroke and acute myocardial infarction, for the people in these said communities. Therefore there should be trained, or training of, health volunteers who can advocated disease awareness and 
promotion of using EMS for transportation into their own language.

\section{Acknowledgement}

We greatly appreciate all the participants, and sincerely acknowledge Dr. Niraj Maskey, language editor.

\section{Funding sources}

This study was funded by the National Research Council of Thailand, and the Center of Excellence for Hilltribe Health Research, Mae Fah Luang University.

\section{Conflict of interest}

This study has no conflict of interest to declare.

\section{References}

1. Jones CM, Wasserman EB, Li T, Amidon A, Abbott M, Shah $\mathrm{MN}$. The effect of older age on EMS use for transportation to an emergency department. Prehosp Disaster Med 2017;32: 261-8.

2. Mercuri M, Connolly K, Natarajan MK, Welsford M, Schwalm JD. Barriers to the use of emergency medical services for ST- elevation myocardial infarction: determining why many patients use for self- transport. J Eval Clin Pract 2018;24:375-9.

3. Wandling MW, Nathens AB, Shapiro MB, Haut ER. Association of prehospital mode of transport with mortality in penetrating trauma: a trauma system-level assessment of private vehicle transportation vs ground emergency medical services. JAMA Surgery 2018;153:107-13.

4. Ponsen K, Wachiradilok $\mathrm{P}$, Sirisamutr $\mathrm{T}$, Doungthipsirikul S. The situation and the factors that affect the use of emergency medical services at the emergency department of patient emergency in Thailand [monograph on the Internet]. Nonthaburi: National Institutes of Emergency Medicine Thailand; 2017 [cited 2019 Mar 1]. Available from: https://www.niems.go.th/th/ Upload/File/255908171439332756_I7UNTXkvtBfQGYyL.pdf

5. Ibanez B, James S, Agewall S, Antunes MJ, Bucciarelli-Ducci $\mathrm{C}$, Bueno $\mathrm{H}$, et al. $2017 \mathrm{ESC}$ guidelines for the management of acute myocardial infarction in patients presenting with ST- segment elevation: the task force for the management of acute myocardial infarction in patients presenting with STsegment elevation of the European Society of Cardiology (ESC). European Heart J 2017;39:119-77.

6. Shatpattananunt B, Wiangosot S, Pintatham K. Elderly patients' experience in using emergency medical service at a tertiary hospital, upper northern Thailand. Songklanagarind J Nurs 2016; 38:102-15.

7. Aringhieri R, Bruni ME, Khodaparasti S, Van Essen JT. Emergency medical services and beyond: addressing new challenges through a wide literature review. Comput Oper Res 2017;78:34968.

8. Akha tribe in Thailand [homepage on the Internet]. Chiang Rai: The Hill Tribe Organization in Thailand; 2015 [cited 2019 Mar 1]. Available from: http://akha.hilltribe.org/thai/

9. Kunstadter P. Ethnicity, socioeconomic characteristics and knowledge, beliefs and attitudes about HIV among Yunnanese Chinese, Hmong, Lahu and Northern Thai in a north-western Thailand border district. Cult Health Sex 2013;15:383-400.

10. Apidechkul $T$, Wongnuch $P$, Sittisarn $S$, Ruanjai $T$. Health situation of Akha hill tribe in Chiang Rai Province, Thailand. J Pub Health Dev 2016;14:77-9.

11. Manotham M, Koychusakun P, Choyae A, Maneerat W, Muangmool J, Vanishprinyakul S. Healthcare knowledge and practice of Akha ethnic group: case study at a Mae-mon Village, Tambon Huay-chom-poo, Amphur Muang, Chiang Rai. Res Health Sci 2018;12:111-20.

12. Khungtumneam K. Ethnography research for nursing service system in ASEA. J Roy Thai Arm Nurs 2014;15:29-35.

13. Seng JS, Lopez WD, Sperlich M, Hamama L, Reed Meldrum $\mathrm{CD}$. Marginalized identities, discrimination burden, and mental health: empirical exploration of an interpersonal-level approach to modeling intersectionality. Soc Sci Med 2012;75:2437-45.

14. Santos Jr, Black AM, Sandelowski M. Timing of translation in cross-language qualitative research. Qual Health Res 2015; 25:134-44.

15. Squires A. Methodological challenges in cross-language qualitative research: a research review. Int J Nurs Stud 2009; 46:277-87.

16. Lincoln YS, Guba E. Naturalistic inquiry. Beverly Hills: Sage; 1985.

17. Sandelowski M, Leeman J. Writing usable qualitative health research findings. Qual Health Res 2012;22:1404-13 
18. Kalich A, Heinemann L, Ghahari S. A scoping review of immigrant experience of health care access barriers in Canada. J Immigr Minor Health 2016;18:697-709.

19. Thummapol O, Barton S, Park T. Healthcare access experiences among indigenous women in northern rural Thailand: a focused ethnographic study. Cent Asian J Glob Health 2018;7:328-43.

20. Apidechkul T, Laingoen O, Suwannaporn S. Inequity in accessing health care service in Thailand in 2015: a case study of the hill tribe people in Mae Fah Luang district, Chiang Rai, Thailand $\mathrm{J}$ Health Res 2016;30:67-71.

21. Mould-Millman NK, Rominski SD, Bogus J, Ginde AA, Zakariah AN, Boatemaah CA, et al. Barriers to accessing emergency medical services in Accra, Ghana: development of a survey instrument and initial application in Ghana. Glob Health Sci Pract 2015;3:577-90.

22. Tate RC, Hodkinson PW, Sussman AL. Lessons learned from the application of mixed methods to an international study of prehospital language barriers. J Mix Methods Res 2017;11: 469-86.

23. Adeleke A, Apidechkul T, Kanthawee P, Suma Y, Wongnuch P,
Pasukphun N. Factors associated with open burning behaviors among Thai and hill tribe farmers in northern Thailand. J Health Res 2017;3:395-402.

24. Kalich A, Heinemann L, Ghahari S. A scoping review of immigrant experience of health care access barriers in Canada. J Immigr Minor Health 2016;18:697-709.

25. Henize AW, Beck AF, Klein MD, Adams M, Kahn RS. A road map to address the social determinants of health through community collaboration. Pediatrics 2015;136:e993-1001.

26. Sultan M, Abebe Y, Tsadik AW, Ababa A, Yesus AG, MouldMillman NK. Trends and barriers of emergency medical service use in Addis Ababa; Ethiopia. BMC Emerg Med 2019; 19:1-8.

27. Sungbun S, Piaseu N, Partiprajak S. Needs of stakeholders in fast-track care for ST-segment elevation myocardial infarction (STEMI). Thai J Nurs Res 2018;32:19-38.

28. Sonkong K, Chaiklieng S, Neave P, Suggaravetsiri P. Factors affecting delay in seeking treatment among malaria patients along Thailand-Myanmar border in Tak province, Thailand. Malar J 2015;14:1-8. 